\title{
Reação Liquenóide oral: relato de caso
}

\author{
Oral Lichenoid reactions: case report
}

Reacción Liquenoide oral- relato de caso

Fernanda Braga Peixoto ${ }^{1}$, Ana Paula Diniz ${ }^{1}$, Diana Pimentel de Melo ${ }^{1}$, Felipe Henrique de Souza Lima ${ }^{1 *}$.

\section{RESUMO}

Introdução: As alterações liquenóides são reações patológicas que se caracterizam como uma resposta imunológica a um agente alergênico como materiais restauradores, medicações sistêmicas ou alimentos, podendo ser observadas em pele e em mucosa. Detalhamento do caso: O objetivo do presente estudo é relatar o caso clínico de uma paciente, CMS, 41 anos diagnosticada com reação liquenóide oral associada a restaurações de amálgama dental e a conduta utilizada para o tratamento desta alteração. $O$ exame complementar de escolha é a biópsia incisional, mas optou-se em substituir a restauração de amálgama para observar se haveria regressão da lesão, sendo uma manobra semiotécnica menos invasiva. Após o período de três meses houve cessão total da lesão e até o presente momento não houve recidivas. Discussão: Por ser diagnóstico diferencial de outras lesões como o líquen plano, faz-se necessário os exames clínicos minuciosos para o correto diagnóstico e tratamento. A remoção do agente causal leva à melhora significativa do quadro.

Palavras-chave: Diagnóstico bucal, Diagnóstico diferencial, Patologia bucal, Erupções liquenóides.

\begin{abstract}
Introduction: Lichenoid changes are pathological reactions that are characterized as an immune response to an allergen agent such as restorative materials, systemic medications or foods. may present on the skin and oral mucosa. Case detailing: The aim of the present study is to report the clinical case of a patient, $41, \mathrm{CMS}$, years old diagnosed with oral lichenoid reaction associated with dental amalgam restorations and the conduct used to treat this change. The complementary exam of choice is the incisional biopsy, but it was chosen to replace the amalgam restoration to see if there was regression of the lesion, being a less invasive semitechnical maneuver. After the period of three months, there was complete regression of the lesion and to date there were no recurrences. Discussion: Because it is a differential diagnosis of other lesions such as lichen planus, accurate clinical examinations are necessary for the correct diagnosis and treatment. Removal of the causative agent leads to significant improvement of the pathological condition.
\end{abstract}

Keywords: Diagnosis oral, Diagnosis differential, Pathology Oral, Lichenoid eruption.

\section{RESUMEN}

Introducción: Los cambios liquenoides son reacciones patológicas que se caracterizan como una respuesta inmune a un agente alergénico, como algunos materiales odontologicos de propósito rehabilitador dental,drogas sistêmicas o alimentos. puede presentarse en la piel y la mucosa oral. Detalle del caso: El objetivo del presente estudio es informar el caso clínico de un paciente de 41 años, CMS, con el diagnóstico de reacción liquenoide oral asociada a restauraciones de amalgama dental y el conducto utiliza para el

${ }^{1}$ Centro Universitário CESMAC, Maceió-AL. * E-mail: felipehrq@hotmail.com 
tratamiento de esta condición. El examen complementario de elección es la biopsia incisional, pero se eligió para reemplazar la restauración de la amalgama para ver si iba a haber regresión de la lesión, siendo una maniobra técnica menos invasiva. Después del período de tres meses, hubo una regresión completa de la lesión y hasta la fecha no hubo recurrencias. Discusión: Debido a que es un diagnóstico diferencial de otras lesiones, como el liquen plano, se requieren exámenes clínicos precisos para el diagnóstico y tratamiento correctos. La eliminación del agente causal conduce a una mejora significativa de la condición patológica.

Palabras-Clave: Diagnóstico Bucal, Diagnóstico diferencial, Patología bucal, Erupciones liquenoides.

\section{INTRODUÇÃO}

As alterações liquenóides são reações patológicas que se caracterizam como uma resposta imunológica a um agente alergênico como materiais restauradores, medicações sistêmicas ou alimentos. Podem ser observadas em pele e em mucosa. Possuem características clínicas e/ou histológica semelhante a outras lesões, sendo o líquen plano o diagnóstico diferencial mais comum (NEVILLE et al., 2009; SANTOS et al., 2017).

A etiologia mais comum das reações liquenóides orais (RLOs) são as restaurações de amálgama. 0 amalgama é um material restaurador dentário consagrado na odontologia e com fortes evidências científicas de sucesso clínico, esse material resulta da mistura de mercúrio, prata, estanho e cobre que são metais responsáveis pela alta resistência mastigatória e durabilidade restauradora desse material. Devido aos avanços das restaurações estéticas em resina composta o amalgama dental vem caindo em decadência, apesar desse fato, o amalgama dental apresenta grande tendência a corrosão e em sua composição há a presença de metais tóxicos, a exemplo do mercúrio, que em alguns casos podem provocar alterações liquenoides na cavidade oral. Essas alterações são de origem inflamatória crônica mucocutanea e podem gerar manifestações de irritação crônica através de hipersensibilidade lenta de contato, o que também junstifica o declínio do uso desse material na odontologia (BERNARDES et al., 2007; BARBOSA et al., 2011; JARDIM et al., 2013; SANTOS et al., 2017).

As RLOs geralmente são assintomáticas, mas em alguns casos os pacientes relatam coceira (prurido), sensação de ardência e dor na região afetada. É uma lesão polimorfa e pode apresentar-se clinicamente como mancha branca, ulcerações generalizadas, estriada, eritomatosa, erosiva ou vesiculosa e ocorre principalmente na mucosa jugal, gengiva e língua (PRADO et al., 1994; BERNARDES et al., 2007; BARBOSA et al., 2011; JARDIM et al., 2013; SANTOS et al., 2017).

O diagnóstico se dá através de achados onde há características clínicas da lesão e a sua relação direta com restaurações de amálgama. Para se confirmar a natureza é feito o exame histopatológico, neste exame é apresentado na maioria das vezes células inflamatórias, prevalecendo as do tipo eosinófilos, neutrófilos, plasmocitos, linfócitos e outras células de origem inflamatória crónica, porém esses padrões celulares são similares a varias doenças e alterações inflamatórias da regão oral, a exemplo principalmente do liquen plano oral. Desta forma o exame histopatológico auxilia na diferenciação das RLOs e outras lesões de origem não inflamatória (BERNARDES et al., 2007; SANTOS et al., 2017; NEVILLE et al., 2009).

O tratamento das RLOs consiste na remoção do fator de irritação, assim a lesão deve desparecer ou diminuir, em casos onde há intensidade dos sintomas, recomenda-se o uso de corticosteroides tópicos ou sistêmicos. Em geral, espera-se o desaparecimento clínico completo da lesão ou o alívio dos sintomas em breve período de tempo, porém em alguns casos a melhora só é observada após um período de três meses (PRADO et al., 1994; BERNARDES et al., 2007; BARBOSA et al., 2011; JARDIM et al., 2013; WERNECK et al., 2016; SANTOS et al., 2017).

Diante do exposto, o trabalho tem por objetivo relatar o caso clínico de uma paciente diagnosticada com reação liquenóide oral associada a restaurações de amálgama e o tratamento proposto onde o mesmo demostrou-se resolutivo para o caso. 


\section{RELATO DE CASO}

Paciente CMS, gênero feminino, 41 anos, leucoderma, compareceu à Clínica Escola de Odontologia do Centro Universitário Cesmac, com a queixa principal "Tem uma mancha branca na minha bochecha e sinto arder". Na história da doença atual, a paciente relatou que o surgimento dos sintomas foi a cerca de dez anos e que havia sintomatologia de dor e ardência da região. Quando questionada, a paciente relatou que nunca teve interesse em descobrir a origem dos sintomas, mas que resolveu procurar tratamento por se tratar de um sintoma provecto que não apresentava melhora mesmo após tanto tempo de seu aparecimento e que o receio de ser algum tipo de câncer bucal levou-a buscar tratamento. Na história médica a mesma afirmou não ter nenhuma alteração sistêmica.

Ao exame extra oral, a paciente apresentou-se dentro dos padrões de normalidade, ao exame intrabucal observou-se na mucosa jugal uma lesão ulcerada, bilateral, de coloração avermelhada, apresentando estriações esbranquiçadas que não saíam ao raspado, verificou-se que a lesão estava em íntimo contato com as superfícies dos dentes 18, 26 e 27 onde havia a presença de restaurações extensas de amálgama dental que se apresentavam escurecidas e indicativas de grande quantidade de mercúrio em sua composição. (Imagem 1. A e B).

Imagem 1 - Lesão bilateral (representado por seta A) em intimo contato com a superfície das restaurações de amalgama dental.
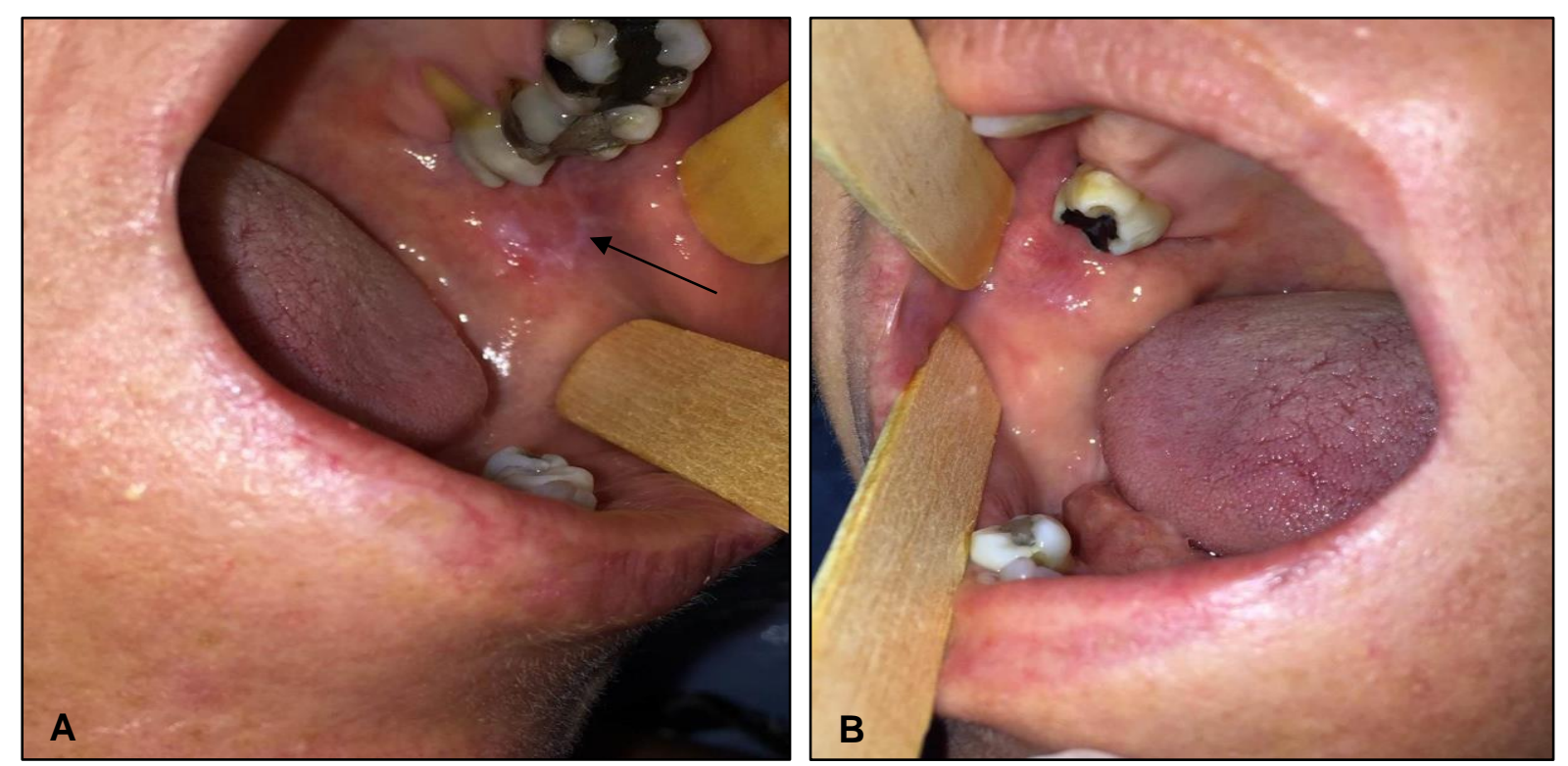

Fonte: Arquivo CESMAC 2017.

As principais hipóteses diagnósticas foram reação liquenóide oral e líquen plano. O exame complementar de escolha é a biópsia incisional, mas neste caso optou-se em substituir a restauração de amálgama para observar se haveria regressão da lesão, sendo uma manobra semiotécnica menos invasiva. Inicialmente foi realizada a remoção do material restaurador em sessão única e a utilização de um material restaurador provisório a base de Oxido de zinco e eugenol (OZE).

Uma semana após a remoção de toda a estrutura da restauração de amalgama houve redução significativa da lesão e da ardência da região (Imagem 2). O material restaurador definitivo de escolha foi a resina composta do tipo micro-híbrida. A paciente foi acompanhada através de exames clínicos quinzenais durante um período de 3 meses onde após esse período foi possível observar a cessão total da lesão. Foi 
indicado o retorno da paciente a Clínica Escola de Odontologia do Centro Universitário Cesmac, após o período de 2 anos para o controle de higiene bucal e manutenção das restaurações substituídas, assim também como a observação de possível recidiva da lesão. Ao retorno foi possível verificar a higienização insatisfatória de toda a região oral, presença de biofilme dental, lesões de cárie, fratura das restaurações, dentre outros problemas dentais ocasionados devido a precária higienização oral deste paciente durante este período. Em relação a lesão confirmou-se cessão total, sem recidivas. A paciente foi instruída e motivada quanto a higiene oral e encaminhada para o setor da disciplina de Estágio Supervisionado em Clínica integrada I, onde a paciente estará sob supervisão e acompanhamento odontológico visando a resolução dos problemas dentários gerados pela higienização oral insatisfatória. (Imagens 3 e 4).

Imagem 2 - Uma semana após remoção do fator alergênico é observado melhora significativa dos sinais clínicos da lesão.

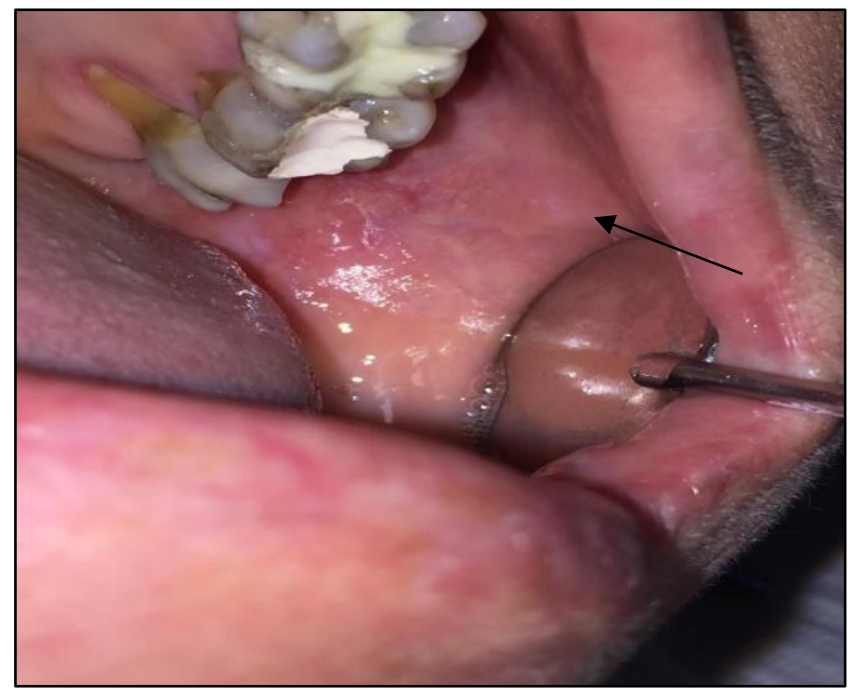

Fonte: Arquivo CESMAC 2017

Imagem 3 - O amalgama dental foi substituído por resina composta.

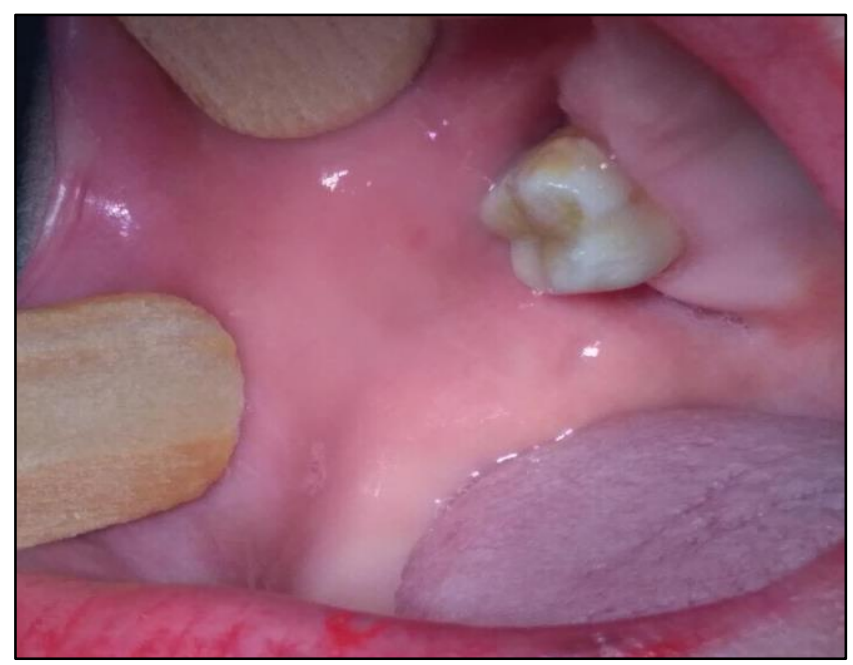

Fonte: Arquivo CESMAC 2017. 
Imagem 4 - Imagem de 2 anos após o tratamento. Observa-se na imagem o descuido com a higiene oral ocasionando a formação de biofilme dental, cárie e fraturas das restaurações. Também é evidente a cessão total da lesão (Representado em seta o local onde se instalava a Reação Liquenóide Oral).

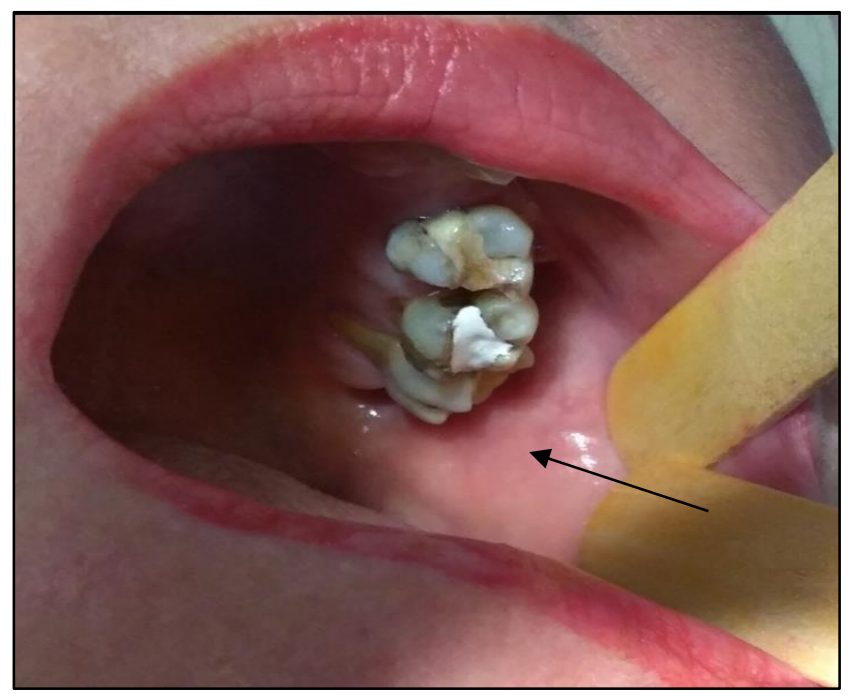

Fonte: Arquivo CESMAC 2017.

\section{DISCUSSÃO}

De acordo com a literatura, a reação liquenóide oral se dá através da alergia a medicamentos, alimentos ou contato da mucosa com metal. No presente caso, o contato direto com o amálgama desencadeou a presença da reação liquenóide oral (BERNARDES et al., 2007; AMARAL et al., 2009; SANTOS et al., 2017).

As lesões liquenóides relacionadas ao amálgama são lesões orais classificadas como relativamente raras e podem gerar sintomas de ardência, prurido (coceira), dor, desconforto na região e sensação de gosto metálico. Clinicamente se apresenta como área ulcerada e/o estriada, geralmente de coloração avermelhada ou esbranquiçada. Sendo assim, para a remissão clinica desta lesão, é necessária a substituição das restaurações de amálgama por restaurações com outro material livre de metal. Nesse caso, a paciente relatou sintomas de ardência e havia a presença de ulcerações e estriações esbranquiçadas em região de mucosa jugal, como forma de tratamento foi adotado um método menos invasivo ao qual preconiza inicialmente a substituição do material restaurador como conduta (BERNARDES et al., 2007; AMARAL et al., 2009; BARBOSA et al., 2011; JARDIM et al., 2013; SANTOS et al., 2017).

Assim como no caso clínico, a literatura mostra que as RLOs tem predileção pelo sexo feminino, aparecem geralmente entre a segunda e quinta década de vida e são frequentemente encontradas em mucosa jugal, gengiva, borda lateral e ventre de língua (BERNARDES et al., 2007; AMARAL et al., 2009; BARBOSA et al., 2011; JARDIM et al., 2013; SANTOS et al., 2017).

O diagnóstico é baseado em alguns aspectos importantes como: A sintomatologia relatada pelo paciente, apresentação clínica da lesão e a aproximação da região lesada com restaurações metálicas. No caso relatado, o diagnóstico foi dado através de uma anamnese cautelosa e achados clínicos característicos da reação liquenóide oral associada ao amálgama dental (BARBOSA et al., 2011). 
O tratamento consiste na remoção dos fatores de irritação local, podendo também ser usada a terapia medicamentosa com corticosteróides nos casos em que há sintomatologia intensa. No caso em questão, foi realizado o tratamento através da remoção do amálgama e substituição por OZE e posteriormente, resina composta. Não houve a necessidade da utilização de medicação para a paciente do caso relatado, pois a mesma não possuía sintomatologia intensa (AMARAL et al., 2009; BARBOSA et al., 2011; JARDIM et al., 2013; WERNECK et al., 2016; SANTOS et al., 2017).

A presença de restaurações de amálgama em contato com a mucosa bucal é um dos fatores que resulta no aparecimento da reação liquenóide oral. Por ser diagnóstico diferencial de outras lesões como o líquen plano faz-se necessário exames clínicos minuciosos para o correto diagnóstico e tratamento desta lesão. A remoção do agente causal leva à melhora significativa do quadro.

\section{REFERÊNCIAS}

1. BARBOSA MO, SILVA AF, CARVALHO RV et al. Lesões liquenóides bucais associadas a restaurações de amálgama: relatos de dois casos. Rev. Odonto Cienc, 2011; 26(3): 258-261.

2. BERNARDES VF, GARCIA BG, SOUTO GR, et al. Lesão liquenóide oral relacionada ao amálgama. An. Bras. Dermatol. 2007; 82(6): 549-52.

3. SANTOS KSS, NETO IJC, PERCIANO CCA et al. REAÇÃO LIQUENÓIDE ORAL ASSOCIADA À RESTAURAÇÕES DE AMÁLGAMA: Relato de Caso. Revista da AcBO,2017; 26(2):49-52.

4. AMARAL SM, MIRANDA AMMA; PIRES FR. Reações medicamentosas na cavidade oral: aspectos relevantes na Estomatologia. Revista Brasileira de Odontologia, 2009, 66(1) 41-53.

5. JARDIM ECG, PERREIRA TTM, SILVA HCL et al. Lichenoid reaction: case report. Brasilian Journal of Surgical and Clinical Research, 2013; 4(1):28-38.

6. NEVILLE BW, DAMM DD, ALLEN CM et al. Patologia oral e maxilofacial. 3.ed. Rio de Janeiro:Elsevier; 2009; $355 p$.

7. PRADO MCP, COSTA LJ, SILVEIRA FRX et al. Reaçöes liquenóides da mucosa bucal. Rev. odontol. Univ São Paulo. 1994; 8(3): 221-4.

8. WERNECK JT, DE MIRANDA FB, JUNIOR AS. Desafios na distinção de lesões de Líquen Plano Oral e ReaçãoLiquenóide. Revistas ,2016; 73(3): 2 